sche Größe - Abstand, Inhalt - derselben verleiht. Nach Behandlung von Punkt, Gerade und Ebene ist ein Kapitel der Kugel, eines den übrigen Flächen zweiter Ordnung und deren Polarentheorie gewidmet. Die Schranbenlinie und flachgängige Schraubenfläche finden knrze Erwähnung; diese heißt wohl zu kurz „di e Schraubenfläche".

A. $L$.

\title{
Das Foucaultsche Pendel und die Theorie der relativen
} Bewegung. Von A. D en izot. Teubner, 1913. Preis geh. M. 3.--

Denizot faßt hier frühere Arbeiten, die in den Berichten der Krakauer Akademie and in den Annalen der Physilk erschienen sind, ergänzend und revidierend zusammen. Er versucht den Nachweis, daß die Vernachlässigung gewisser Glieder, welche in den Differentialgleichungen der an der Erdoberfiäche stattfindenden Bewegung eines Körpers vorkommen und das Quadrat der Winkelgeschwindigkeit der Erde enthalten, vollständig unbegründet sei und daß ferner die in den Lehrbüchern der analytischen Mechanik sich vorfindende Rechnung zur Interpretation des Foucaultschen Pendelversuches nicht aufrecht erhalten werden könne. Denn ein Glied in den allgemeinen Differentialgleichungen wurde bisher nicht richtig aufgefaßt nnd unbegründeterweise als klein vernachlässigt. Nach Denizot kann nun gerade dieses Glied zur Erklärung des Sinusgesetzes dienen.

Der größeren Übersichtlichkeit halber ist die veltoranalytische Darstellung gewählt.

Wilhelm Michl.

Hervorragende Leistungen der Technik. Von K. Schreber. I. Teil (Bd. 20 von Prof. Dr. Bastian Schmids naturwissenschaftlicher Schülerbibliothek), Teubner, 1913, geb. I. 3.-.

Mit außergewöhnlichem didaktischen Geschick begabt, versteht es der Verfasser, dem Leser ein lebendiges Bild von den Methoden und Resultaten moderner Technik za geben. Von besonderem Wert ist der Umstand, daß die Technik als Anwendung der Physik dargestellt wird, wodurch sich Gelegenheit zu einer ebenso gründlichen als interessanten Erörterung der physikalischen Begriffe und Gesetze bietet. Das vorliegende Bändchen behandelt das Gebiet der Mechanik und Kalorik. Ein folgendes soll der Optik und Elektrik gewidmet sein. Im ersten Kapitel (über den Brückenbau) werden die Gesetze der Elastizität und der Kräfteznsammensetzung, im zweiten (über Wind- und Wasserkräfte) die Begriffe von Kraft, Masse und Arbeit und im dritten (über Wärmekraftmaschinen und Heizung) die Grundzüge der Thermodynamik behandelt. Überall wird auch das historische Moment berücksichtigt. Die Abbildungen sind mustergültig.

Der Phänomenalismus. Eine naturwissenschaftliche Weltanschauung von Dr. Hans Klein peter. Leipzig 1913. Joh. Ambr. Barth. 285 Seiten.

In der letzten Zeit hat sich, besonders unter dem Einfluß ron Mach, eine vielen Naturforschern gemeinsame philosophische Grundüberzeugung ausgebildet, die bestrebt ist, ohne jedes Zurückgehen auf empirisch nicht nachweisbare Realitäten, das ganze Weltbild nur aus den Sinnesempfindungen aufzubauen. Neben dem uns unmittelbar gegebenen, den "Phänomenen", die unserer Willkür nicht unterworfen sind, gibt es nach dieser Auffassung noch eine 